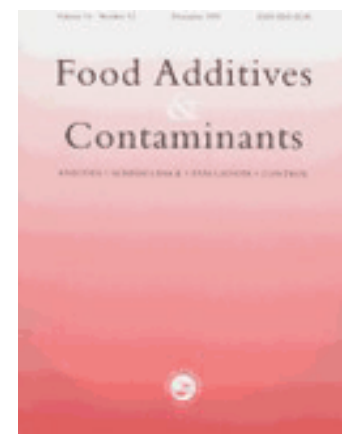

\title{
Estimate of intake of benzoic acid in the Belgian adult population
}

\begin{tabular}{|r|l|}
\hline Journal: & Food Additives and Contaminants \\
\hline Manuscript ID: & TFAC-2008-373.R1 \\
\hline Manuscript Type: & Original Research Paper \\
\hline Author: & $24-$ Seb-2009 \\
\hline Complete List of Authors: & $\begin{array}{l}\text { Vandevijvere, Stefanie; Scientific Institute of Public Health, } \\
\text { Epidemiology } \\
\text { Andjelkovic, Mirjana; Scientific Institute of Public Health, Food } \\
\text { De Wil, Marc; Scientific Institute of Public Health, Food } \\
\text { Vinkx, Christine; Federal Service of Public Health, Food Chain and } \\
\text { the Environment } \\
\text { Huybrechts, Inge; Ghent University, Public health } \\
\text { Van Loco, Joris; Scientific Institute of Public Health, Food } \\
\text { van oyen, herman; Scientific Institute of Public Health, } \\
\text { Epidemiology } \\
\text { Goeyens, Leo; Scientific Institute of Public Health, Food }\end{array}$ \\
\hline Methods/Techniques: & $\begin{array}{l}\text { Chromatographic analysis, Chromatography - HPLC, Exposure } \\
\text { assessment }\end{array}$ \\
\hline Additives/Contaminants: & Additives general \\
\hline Food Types: & Beverages, Confectionary, Fish and fish products, Snack products \\
\hline &
\end{tabular}

\section{SCHOLARONE Manuscripts}




\title{
1 Estimate of intake of benzoic acid for the Belgian adult population
}

Vandevijvere S. ${ }^{1}$, Andjelkovic M. ${ }^{2}$, De Wil M. ${ }^{2}$, Vinkx C. ${ }^{3}$, Huybrechts I. $^{4}$, Van Loco J. ${ }^{2}$, , Van Oyen H. ${ }^{1}$, Goeyens, L. ${ }^{2}$

${ }^{1}$ Scientific Institute of Public Health, Unit of Epidemiology, Brussels, Belgium

${ }^{2}$ Scientific Institute of Public Health, Unit of Food, Brussels, Belgium

${ }^{3}$ Federal Public Service of Health, Food Chain Safety and Environment, Brussels, Belgium

${ }^{4}$ Department of Public Health, Ghent University, Ghent, Belgium

\begin{abstract}
An exposure assessment was performed to estimate average daily benzoic acid intake for Belgian adults. Food consumption data were retrieved from the national food consumption survey. In a first step, individual food consumption data were multiplied with the maximum permitted use levels for benzoic acid per food group (Tier 2). In a second step, a label survey to identify the foods where benzoic acid is effectively used as an additive and a literature review of the possible occurrence of benzoic acid as a natural substance, were performed. With this information a refined list of foods was drafted for the quantification of benzoic acid, which was performed by an HPLC method, optimized and validated for this purpose. Individual food consumption data were then multiplied with the actual average concentrations of benzoic acid per food group (Tier 3). Usual intakes were calculated using the Nusser method. The mean benzoic acid intake was $1.58 \mathrm{mg} / \mathrm{kg}$ bw/day (Tier 2) and $1.25 \mathrm{mg} / \mathrm{kg}$ bw/day (Tier 3). In Tier 2, men exceeded the acceptable daily intake (ADI) of $5 \mathrm{mg} / \mathrm{kg}$ bw/day at the $99^{\text {th }}$ percentile. The greatest contributors to the benzoic acid intake were soft drinks. Benzoic acid as a natural substance represents only a small percentage of the total intake. The results show that actual benzoic acid intake is very likely to be below the ADI. However, there is a need to collect national food consumption data for children as they might be more vulnerable for an excessive intake.
\end{abstract}




\section{Introduction}

Benzoic acid (E210) or its sodium (E211), potassium (E212) or calcium (E213) salt, is intentionally added to certain foods as a food preservative or may naturally be present in specific foods. Sodium benzoate is about 200 times more soluble than benzoic acid and is used preferably as one of the main antimicrobial preservatives in foods and beverages. Benzoic acid is used as an antiseptic, antimycotic and antipyretic agent (Suhr and Nielsen 2004).

The temporary acceptable daily intake (ADI) was set at $5 \mathrm{mg} / \mathrm{kg}$ body weight by the European Scientific Committee on Food in 1994. The Joint WHO/FAO Expert Committee on Food Additives (JECFA) set an ADI of $5 \mathrm{mg} / \mathrm{kg}$ body weight in 1996, which was a confirmation of the evaluation in 1994. Use of benzoic acid is authorised in the European Union in several food groups on the condition of respecting the maximum use levels (Directive 95/2/EC), which are all expressed as the free acid. The European Commission (Directive 95/2/EC) asks the Member States to monitor the consumption and use of food additives, in order to allow evaluation of the consumption trends as well as differences in consumption patterns among various Member States.

The Report from the European Commission on Dietary Food Additive Intake in the European Union (European Commission 2001) describes a harmonized, tiered method to estimate additive intakes. In Tier 1, the theoretical food consumption data are combined with the maximum permitted usage levels for the additive. This conservative estimate was done at the EU level and is not a task for the Member States, as no national data are used. Only for a limited list of additives, national estimates are necessary to refine the estimate for those additives for which in Tier 1 an exceeding of the ADI was observed. In the next step (Tier 2), actual national food consumption data are used and combined with maximum permitted usage levels. Such intake estimate will still overestimate the real intake, but can exclude additives which do not pose a major risk from the most difficult estimate in Tier 3: a combination of actual national food consumption data with actual usage levels of the additive.

A study on the intake of benzoic acid was chosen because of the following elements: (1) high intakes were estimated in other countries; (2) benzoic acid is much less reactive than sulphites or nitrites resulting in residual levels more close to usage levels; (3) feasibility to analyse; (4) benzoic acid is important in soft drinks for which consumption data are high and increasing; (5) there is a long list of food groups in which the additive is authorised and extensions of authorisations are from time to time requested.

In a former study in Belgium, a Tier 2 exposure assessment of benzoic acid was carried out in three 
record), adolescents from a major city in Flanders, Ghent ( $n=341$; age 14-18; 7-day estimated dietary record) and women of reproductive age from Flanders (n=641; age 18-40; 2-day estimated dietary record) (Bilau et al. 2008). The median estimated daily intake was respectively $2.0 \mathrm{mg} / \mathrm{kg}$ bw, 1.7 $\mathrm{mg} / \mathrm{kg}$ bw and $1.92 \mathrm{mg} / \mathrm{kg}$ bw. In all age groups the greatest contributor to total benzoic acid intake was the group of non-alcoholic flavoured drinks. At respectively the $92^{\text {nd }}$ percentile (children), the $97^{\text {th }}$ percentile (adolescents) and the $94^{\text {th }}$ percentile (women), the ADI was slightly exceeded. This study showed the need to perform a more refined estimate, using actual levels.

Besides being widely used as a food additive, benzoic acid occurs naturally in different fruits 85 (Hegnaur 1992). Appreciable amounts have been detected in most berries (around 0.05\%) (Budavari et al. 1996), in particular in cranberries where they are present in a range from 300 to $1300 \mathrm{mg} / \mathrm{kg}$ (Hegnaur 1966). Benzoic acid has also been detected in many animal products, including dairy products, as the result of the lactic acid fermentation (Sauh et al. 1990). It is also produced during the ripening of cheese by the propionic acid fermentation following the lactic acid fermentation (Thierry and Maillard 2002). Naturally occurring benzoic acid has also been found in honey (Steeg and Montag 1987), mushrooms (Beltran-Garcia 1997) and wine (Matejicek et al. 2003). Further, benzoic acid can be used as a plasticizer in the manufacturing of plastic packing materials for yoghurt, butter or margarine (Vermeiren et al. 2002). The latter source of contamination with benzoic acid will not be further discussed in this paper.

The objective of this study was to perform a refined estimate of the benzoic acid intake in the Belgian adult population, using the data from the national food consumption survey of 2004.

100 obtained for chosen food groups, and then, as far as possible, actual average levels of benzoic

101 acid were combined with consumption data to perform a Tier 3 exposure assessment. Besides

102 taking into account additive use, the refined intake estimate also took into account the natural 103 presence of benzoic acid in certain foods to obtain the best estimate of real intake. In Tier2, as 104 well as in Tier 3, intake of benzoic acid was compared with the ADI,

\section{Materials and methods}

106

\section{Study design}

108 For the Tier 2 exposure assessment, maximum usage levels of benzoic acid in different foods were as 109 far as possible linked to the food consumption data of these specific foods, derived from the Belgian 110 food consumption survey 2004). For some foods in the EU legislation, it is unlikely that they exist on 
111 the Belgian market (for example grape juice for sacramental use or liquid tea concentrates). Other

112 products were not found in the food consumption survey (e.g. food supplements). For the authorised

113 quantum satis use as surface treatment of dried meat products, a Tier 2 approach was not possible. The

114 exclusion of some food groups could lead to an underestimation of the intake, but this was supposed to 115 be very limited.

116

117 For the Tier 3 exposure assessment, first a food label survey was performed and then samples of 118 different foods, in which benzoic acid is actually added or present as a natural substance, were taken 119 from different supermarkets. In these samples concentrations of benzoic acid were determined with an 120 optimized and validated analytical method. Benzoic acid concentrations in different foods were then 121 linked to the consumption quantity of these foods, calculated from the data of the food consumption 122 survey. For foods for which no analyses were performed (e.g. shrimps, crustaceans and molluscs, 123 which were designated as a less important source of benzoic acid due to its less frequent intake), the 124 maximum permitted level of benzoic acid was used.

\section{Food consumption data}

127 Consumption data from the national food consumption survey 2004 were used to perform the exposure 128 assessments. Aims, design and methods of this survey are described elsewhere (De Vriese et al. 2005). 129 The target population comprised all Belgian inhabitants of 15 years or older. The sample included 1303245 participants randomly selected from the National Register, using a multi-stage stratified 131 procedure.

132

133 Information on dietary intake was collected by two non-consecutive 24-hour recalls in combination 134 with a food frequency questionnaire. During the 24-hour recall interviews the respondent reported the 135 quantity of all foods and beverages consumed during the preceding day. The 24-hour recall was 136 carried out using EPIC-SOFT software (Slimani and Valsta 2002). This program allows obtaining very 137 detailed information about the foods consumed and the recipes used in a standardized way. 138

1393083 participants completed two 24-hour recalls of which 1537 women and 1546 men. Participants 140 were categorized into four age groups: $15-18$ years $(n=760), 19-59$ years $(n=830), 60-74$ years $(n=789)$ 141 and 75 years or older $(n=704)$.

\section{Benzoic acid concentration of selected foods}

144 Before the development of the sampling plan for the collection of the samples to be analysed, a food 145 label survey was performed to identify the food products where benzoic acid is actually used as an 146 additive. Five supermarket chains with good market share in Belgium were visited. Based on the 147 regulation (Directive 95/2/EC), several food groups have been surveyed, 1245 items in total. Products 
148 were selected according to the indications on the labels concerning addition of benzoic acid. Although 149 this was not an absolutely exhaustive survey of all existing categories of food, it was attempted to 150 cover the most important national and marketplace's brands. The final goal of the survey was to get an 151 overview of the general manufacturing practices regarding benzoic acid application as an additive in 152 Belgium.

154 Further, a literature review of the possible occurrence of benzoic acid as a natural substance was 155 performed. After this first selection of foods, a refined list of foods was drafted for the quantification 156 of benzoic acid. The food consumption survey 2004 was used to evaluate the various channels used 157 for food provisions (supermarkets), the frequencies of consumption of different foods and the 158 preparations of foods. The Tier 2 exposure assessment provided an indicative list of the most 159 important foods contributing to benzoic acid intake in the Belgian population. The comparison of the 160 Tier 2 acquired list with the refined list from the label survey and knowledge of foods in which 161 benzoic acid may be present as a natural substance, permitted the development of the sampling plan 162 for the collection of the food samples to be analysed.

164 A great effort was put into ensuring representative concentration data of benzoic acid in foods on the 165 Belgian market. The sampling plan included two categories: food items with benzoic acid used as an 166 additive and those where benzoic acid is likely to be present as natural substance. In total for both 167 categories 58 samples ( 28 and 30 , respectively to the category) were collected and analysed. The 168 results of the analyses were grouped to ensure a clear data presentation. The grouping was performed 169 according to the EU directive 95/2/EC as follows: desserts, confectionary, prepared salads, non 170 emulsified sauces, and non-alcoholic drinks. Additionally, two food groups were included, namely 171 dairy products (cheese, yoghurt, and dairy flavoured with fruits) and products based on fruits (berries, 172 grapes, apples, pears and tropical fruits). Collection of samples was performed from August to 173 September 2007 in 5 major supermarket chains with a good market share. These 5 food distributors 174 are visited by approximately $75 \%$ of the Belgian population in search for their food provisioning 175 (CRIOC - OIVO 2007). Prepared salads were additionally taken from sandwich delivery snack bars. If 176 the analyses were not conducted on the same day, the sample was stored deep frozen for a maximum 177 time of one month.

179 Extraction from and determination of benzoic acid in different matrices was done by a modified 180 method described in ISO/FDIS 22855:2007(E) (International Organisation for Standardisation 2007) 181 where benzoic acid was quantified by a high performance liquid chromatography method optimized 182 and validated for this purpose. This proposed method is based on a formerly used method (Küppers 183 and Jans 1988). 
185 Sample preparation and extraction of benzoic acid was adjusted to the state (liquid or solid) of the

186 sample. In this manner liquid samples were homogenised prior to opening of the package while 187 carbonated drinks were degassed in an ultrasonic bath for 3-5 min. A sample (10ml) was transferred 188 into a $50 \mathrm{ml}$ conical tube where $10 \mathrm{ml}$ of methanol-water $(80: 20, \mathrm{v}: \mathrm{v})$ solution of 3-chlorobenzoic acid 189 (75mg/l) was added. The solution $\mathrm{pH}$ was adjusted with a droplet of $36.5 \% \mathrm{HCl}$. In the following step 190 the solution was vortex mixed and centrifuged for 5 minutes at 7000rpm. From this $1 \mathrm{ml}$ was used for 191 further purification.

192

193 Solid samples (200g) were homogenised with an Ultra-Turrax mixer. Out of that $10 \mathrm{~g} \pm 0.1 \mathrm{~g}$ was 194 weighed and transferred into a 50ml conical tube. $10 \mathrm{ml}$ of methanol-water (80:20, v:v) solution of 3195 chlorobenzoic acid $(75 \mathrm{mg} / \mathrm{l})$ was added. After adding a droplet of $36.5 \% \mathrm{HCl}$, the tube was closed and 196 vortex mixed (non fatty solid partially hydrophilic samples, e.g. jams and gums) or mixed with the 197 Ultra-Turrax (fatty solid hydrophobic samples, e.g. cheese, fish, and crustaceans) until dissolution into 198 the aqueous phase was obtained. If necessary, the sample was placed in a warm $\left(45-60^{\circ} \mathrm{C}\right)$ water bath 199 for $30 \mathrm{~min}$ with frequent agitation. In the following step the solution was vortex mixed, centrifuged 200 for $5 \mathrm{~min}$ at $7000 \mathrm{rpm}$ and cooled to room temperature. From this $1 \mathrm{ml}$ was used for further 201 purification.

202

203 All prepared samples were subjected to purification through SPE C18ec (Chromabond, 500mg, 3ml) 204 which was conditioned by 1 cartridge volume methanol followed by 1 cartridge volume $\mathrm{HCl} 0.1 \mathrm{M}$. 205 Next, $1 \mathrm{ml}$ of the sample was applied (at the flow rate $0.4 \mathrm{ml} / \mathrm{min}$ ) and the cartridge was successively 206 washed twice with $2 \mathrm{ml}$ of $0.1 \mathrm{M} \mathrm{HCl}$. and additionally washed with $1 \mathrm{ml}$ hexane where before and after 207 this step the cartridge was left to dry for 3 or $1 \mathrm{~min}$ (respectively). The extract was eluted with $3 \mathrm{ml}$ 208 methanol and concentrated under nitrogen at $40^{\circ} \mathrm{C}$ to approximately $1 \mathrm{ml}$.

210 Finally the extracts were filtered through PTFE $0.45 \mu \mathrm{m}$ and injected into HPLC. HPLC analysis was 211 conducted using an Agilent 1100 Series (Waldbronn, Germany) equipped with a quaternary pump, an 212 auto sampler and DAD detector operated by ChemStation software (version B.01.03) A LiChrospher 213 RP Select B (5 $\mu \mathrm{m} ; 250 \times 4.6 \mathrm{~mm})$ HPLC column with Lichrospher RP Select B pre-column was used 214 throughout the study.

216 The separation was obtained by gradient elution with three mobile phases. Mobile phase A 217 (ammonium acetate aqueous solution, $\mathrm{pH} 3.9,0.05 \mathrm{M}$ ) was kept constant at 10\% while mobile phase $\mathrm{B}$ 218 (acetonitrile) and C (MiliQ water) were kept constant first $10 \mathrm{~min}: 25 \% \mathrm{~B}$ and $65 \% \mathrm{C}$; then the 219 gradient changed $10-13 \min 60 \% \mathrm{~B}$ and $30 \% \mathrm{C}$ and kept constant for $7 \mathrm{~min} ; 20-21 \mathrm{~min}$ : $25 \% \mathrm{~B}$ and 
$65 \% \mathrm{C}$ and the system was equilibrated for $4 \mathrm{~min}$. The flow rate was $1 \mathrm{ml} / \mathrm{min}$ at $35^{\circ} \mathrm{C}$. The detection was carried out by DAD detector at 235 and $255 \mathrm{~nm}$.

Identification of benzoic acid was done by comparing the retention time and peak shape to that of reference standard whereas quantification was performed according to the calibration curve $\left(y=1.48 x+0.03 ; R^{2}=0.99\right)$ plotted as the respond ratio of benzoic acid and 3-chlorobenzoic acid versus concentration of benzoic acid in the range $0-250 \mathrm{mg} / \mathrm{l}$ for nine calibration points $(0 ; 0.2 ; 0.5 ; 1 ; 5 ; 10$; $25 ; 50 ; 100 ; 250 \mathrm{mg} / \mathrm{l})$.

The method was validated on 3 different matrices: sugared liquids, emulsified solids and dairy products (mayonnaise and plain yoghurt respectively). The limit of detection (LOD) and limit of quantification (LOQ) for benzoic acid were 2.5 and $5 \mathrm{mg} / \mathrm{l}$ respectively. The intra-day and inter-day precision of the method showed a coefficient of variation $(\% \mathrm{CV})$ between 0.25 and $16 \%$ for spiked samples (taking $\leq 10 \%$ as acceptable limit) and the recovery of the method was found to be between 87 to $113 \%$.. The precision of the method was dependent on the matrix and the concentration. For both non-alcoholic flavoured drinks (soft drinks) and dairy products (yoghurt) the lowest level (10 mg/l and $5 \mathrm{mg} / \mathrm{kg}$ respectively) resulted in higher \%CV's (12-16\%) whereas CV's for determination of benzoic acid in fatty samples (mayonnaise) were low at all three levels.

\section{Statistical analysis}

Only respondents with two completed 24-hour recall interviews were included in the analyses $(\mathrm{n}=$ $3083 ; 1546$ men and 1537 women).

The individual intake of benzoic acid from a certain food product was estimated by the following equation:

$y_{i}(m g / k g b w / d a y)=\frac{c_{i} \times x_{i}}{b w_{i}}$,

where $y_{i}$ is the intake of benzoic acid by individual $i$ from a particular food (in mg per kg bodyweight per interview day), $c_{i}$ is the concentration of benzoic acid in that food ( $\mathrm{mg}$ per $\mathrm{kg}$ ), $x_{i}$ is the consumption of a certain food by individual $i(\mathrm{~kg})$ and $b w_{i}$ is the self-reported body weight of individual $i(\mathrm{~kg})$. To estimate the total intake of benzoic acid per food group or per day, individual daily intakes of benzoic acid from different foods were added up.

As mentioned before, two approaches were used for the calculation of benzoic acid intake. In the Tier 2 approach, $c_{i}$ represents the maximum allowed concentration in each food. The maximum permitted levels of benzoic acid used in different foods are listed in the European Directive 95/2/EC (Table 1) ( 
253 Directive 95/2/EC). In the Tier 3 approach, $c_{i}$ represents the actual average concentration of benzoic 254 acid that is observed in a particular food.

256 The usual intake distribution for benzoic acid was estimated with the Nusser method (Nusser et al. 257 1996) using the C-side software (Iowa State University 1996). Several statistical methods are available 258 to estimate usual intake distributions with the correct mean, variance and skewness. These statistical 259 procedures adjust for within-person or day-to-day variability. Of all different statistical procedures, the 260 Nusser method (Nusser et al., 1996) is highly recommended because it eliminates the intra-individual 261 variance and additionally transforms the data to obtain approximately normally distributed data. The 262 method is suitable to estimate usual intake distributions in a population both for normally and non263 normally distributed foods and nutrients. The usual intake distribution was weighted and adjusted for 264 the age and sex distribution of the Belgian population and adjusted for day of the week and season. 265

\section{Results}

The label survey revealed that in biscuits, cakes and confectionary no benzoic acid is used on the 269 Belgian market, although it is allowed. In the category of emulsified sauces the difference between regular and light sauces was remarkable. Of 55 light emulsified sauces, 19 contained benzoic acid (35\%) whereas only $10 \%$ of the regular products had the additive indicated on the label. From the results it appeared that benzoic acid may more often be used in light sauces. Similarly, in relation to the fat percentage of the product, it was observed that benzoic acid was more often used in products containing less than $60 \%$ fat.

The concentration of benzoic acid in samples suspected to contain benzoic acid as a natural substance, was under official limits. Red and white wine, fruit juices and syrups, in particular those from berries, did not contain benzoic acid above the LOQ. Among dairy products, the highest amount of benzoic 280 acid was detected in cottage cheese. The average amounts in yoghurts were around $25 \mathrm{mg} / \mathrm{kg}$ which was comparable to the amounts detected in different yoghurts collected from stores in Belgium in 1982 (Guyot et al. 1998; Sieber et al. 1995; Van Renterghem 1982). On the other hand, the amount of the additive in all analysed soft drinks (coke and lemonade) was between $100 \mathrm{mg} / \mathrm{l}$ and the maximum permitted level of $150 \mathrm{mg} / \mathrm{l}$. As a result, the use of actual levels instead of maximum levels did not reduce the intake estimate dramatically (Table 1). Intake estimates of consumption of food groups can

287 The Tier 2 approach, which is the most conservative approach, revealed a mean usual daily benzoic 288 acid intake of $1.58 \mathrm{mg} / \mathrm{kg}$ bw/day (Table 3; Figure 1). At the $97.5^{\text {th }}$ percentile, the usual intake of 289 benzoic acid was $3.97 \mathrm{mg} / \mathrm{kg}$ bw/day which was still below the ADI. The most important contributor 
to the intake of benzoic acid was the group of non-alcoholic flavoured beverages (31.07\%). Other important contributors at the upper end of the intake distribution were non-emulsified sauces $(16.16 \%)$, vegetables in vinegar, brine or oil $(13.24 \%)$, prepared salads $(8.08 \%)$, semi-preserved fish products $(6.90 \%)$ and shrimps, molluscs and crustaceans $(6.84 \%)$.

The Tier 3 approach is more accurate than the Tier 2 since it takes into account the actual average concentrations of benzoic acid in different foods and it also considers the presence of benzoic acid as a natural substance. Nevertheless the results of Tier 3 were comparable to those of Tier 2 (Table 4, Figure 2), where no exceeding of the ADI was observed. Non-alcoholic flavoured drinks (34.28\%) like coke and lemonade were again the most important contributors to the intake of benzoic acid. Benzoic acid present as a natural substance in food only contributed to a very small percentage (2.7\%) to the total benzoic acid intake (Table 4).

It was observed that the benzoic acid intake in men was higher than in women (Figure 1; Figure 2). Mean usual benzoic acid intake in men (Tier 3) was $1.41 \mathrm{mg} / \mathrm{kg}$ bw/day. For women mean usual benzoic acid intake (Tier 3) was $1.22 \mathrm{mg} / \mathrm{kg}$ bw/day. Men consumed more non-alcoholic flavoured beverages than women $(288 \pm 307 \mathrm{~g} /$ day versus $177 \pm 198 \mathrm{~g} /$ day for women $)$, which were the main contributors to the benzoic acid intake. Men only exceeded the acceptable daily intake at the $99^{\text {th }}$ percentile for the Tier 2 estimation at an intake of $5.18 \mathrm{mg} / \mathrm{kg}$ bw/day (while women only reached $4.22 \mathrm{mg} / \mathrm{kg}$ bw/day at the $99^{\text {th }}$ percentile). However, men did not exceed the ADI at the $99^{\text {th }}$ percentile for the Tier 3 estimation where the intake was $4.64 \mathrm{mg} / \mathrm{kg}$ bw/day (while $4.10 \mathrm{mg} / \mathrm{kg}$ bw/day for women).

\section{Discussion}

The food label survey showed that in some products, like confectionary, although allowed, no benzoic acid is used in Belgium. Remarkable was that benzoic acid is used more frequently in light sauces than in regular sauces. A possible explanation could be the higher water content of these products which in turn require higher levels of benzoic acid to exert a sufficient preservative effect. The analyses of the food samples for quantification of benzoic acid showed that no products with higher levels of benzoic acid than allowed were present in selected food stores in Belgium.

In the most conservative method (Tier 2), both mean usual benzoic acid intake and the usual benzoic acid intake at the higher percentiles were found to be lower than the ADI. When a Tier 3 exposure assessment was performed, the mean usual intake of benzoic acid and the usual intake of benzoic acid at the higher percentiles were also lower than the ADI, which means there is no direct concern of an excessive benzoic acid intake in the Belgian adult population. Benzoic acid intake in this population 
327 may be regarded as safe and does not need further consideration if dietary patterns do not change

328 tremendously. However, there are some minor concerns which may be raised.

330 The most important contributor to the benzoic acid intake in the Belgian adult population is the food 331 group of non-alcoholic flavoured drinks. People might be loyal to one brand and hence not consume 332 an average concentration. Using maximum levels in the calculation might better protect consumers. As 333 explained above, some food groups in which use of benzoic acid is authorised were not taken into 334 account. Drugs and cosmetics were not taken into account either.

336 In the Belgian population 15 years and older, there is no direct threat to consumers regarding benzoic 337 acid intake. However, there may be special groups which are at risk for exceeding the ADI. First, men 338 seem to have a higher intake of benzoic acid than women, due to a higher intake of soft drinks. Men 339 exceed the ADI at the $99^{\text {th }}$ percentile in a Tier 2 exposure assessment. Second, soft drinks are usually 340 related to children's preferences and children are particularly vulnerable to food safety issues. On the 341 other hand sodium benzoate will be phased out as additive in soft drinks, resulting in lower intake of 342 benzoic acid in the near future (Nyman et al. 2008). Moreover it is remarkable that no benzoic acid is 343 used in Belgium in cakes, biscuits and confectionary, food groups which are frequently consumed by 344 children.

346 Unfortunately, the lack of national data about children's consumption habits posed a limitation to the 347 study. The EU Scientific committee on Food has recommended that special attention should be given 348 to additive intake in children, since the intake is expressed on a body weight basis and may be 349 markedly higher than that of adults.

351 Benzoic acid has also been linked to hyperactivity in children when mixed with other artificial food 352 colours (McCann et al., 2007). In Flemish preschool children, the calculated (Tier 2) intake estimate 353 was only slightly higher than in adolescents or adult women (Bilau et al., 2008).

355 The intake as well as the pattern of benzoic acid in the Belgian population was comparable with that in 356 some other countries but somewhat higher. The average intake of benzoic acid in Australia and New 357 Zealand was between 10 and $15 \%$ of the ADI for the consumers older than 2 years whereas for the $95^{\text {th }}$ 358 percentile the intake was between $50 \%$ and $65 \%$ of the ADI. The most exposed population group were 359 children between 2 and 12 years. In this age group, benzoic acid exposure was estimated to be about 360 1.2-1.4 times the ADI (with higher intake for boys than for girls). The major intake for the average 361 population came from soft drinks (55\%), regular cordial (21\%) and orange juice (18\%) (Food 362 Standards Australia New Zealand 2005). 
364 An intake survey of different food additives in Finland (Pentilla et al. 1988) identified fruit and berry 365 juices, soft drinks and semi-preserved fish as the major sources of intake of benzoic acid which was 366 estimated to be $40 \mathrm{mg} /$ day representing $13 \%$ of the acceptable daily intake. Those results were 367 obtained from the food balance sheets of the Government statistical office and actual concentration in 3681400 foods products was used.

At last, there are some important methodological limitations that should be taken into account when interpreting the findings of this study. First, it is very difficult to exactly match the food groups or 372 items defined in the legislation with the food groups or items defined in the food consumption survey. 373 Secondly, some potentially important food groups, for example liquid eggs used in industrial 374 processes, to which benzoic acid is commonly added as a preservative, were neglected in this study. It 375 was not possible to disaggregate food consumption data up to this level. Further, benzoic acid in 376 seasonings and condiments and in grape juice fermented for sacramental juice was neglected because 377 of an assumed low consumption and a difficult match with food consumption data.

In conclusion, the intake of benzoic acid in the Belgian adult population does not imply an immediate 380 threat to consumers. Nevertheless, the intake is close to the accepted daily intake level and this 381 maximum level may be exceeded if dietary habits change for example when soft drink consumption 382 increases or when authorisations are extended. No data about children are available at this moment 383 while children are considered the most vulnerable population group. In the future, a food consumption 384 monitoring system should be organised in Belgium to be able to evaluate additive intakes of different 385 population groups on a regular basis.

\section{Acknowledgements}

The authors declare not having any conflicts of interest. The authors acknowledge the work of the 390 field work team of the food consumption survey: Stéphanie De Vriese and Michel Moreau and the 391 dietary support from Mia Bellemans, Mieke De Maeyer, Kadija El Moumni and Davy Van Steenkiste. 392 Special thanks go to the respondents and the dieticians during the field work. The members of the 393 steering committee (Leen Rasschaert and Charles Cremer) are greatly acknowledged for their 394 scientific advice. The survey was funded by the Federal Ministry of Health, Food Chain and 395 Environment.

\section{References}


403 Beltran-Garcia MJ. 1997. Volatile compounds secreted by the Oyster Mushroom (Pleurotus 404 ostreatus) and their antimicrobial activities. Journal of Agricultural and Food Chemistry 45: 405 4049-4052.

406 Bilau M, Matthys C, Vinkx C, de Henauw S. 2008. Intake assessment for benzoates in 407 different subgroups of the Flemish population. Food Chem Toxicol 46: 717-723.

408 Budavari S, O'Neil MJ, Smith A, Heckelman PE, Kinneary JF. 1996. The Merck index -- an 409 encyclopedia of chemicals, drugs and biologicals. Merck\&Co Inc., New York.

410 CRIOC - OIVO. 2007. Canaux de distribution des produits alimentaires. Centre de Recherche 411 et d'Information des organisations de consommateurs (CRIOC)

412 De Vriese S, Debacker G, de Henauw S, Huybrechts I, Kornitzer M, Leveque A, Moreau M, 413 Van Oyen H. 2005. The Belgian food consumption survey: aims, design and methods. Arch 414 Public Health 63: 1-16.

415 European Commission. 2001. Report from the Commission on dietary food additive intake in 416 the European Union. European Commission, Brussels.

417 Food Standards Australia New Zealand. 2005. The 21st Australian Total Diet Study. A total 418 diet study of sulphites, benzoates and sorbates. Food Standards Australia New Zealand

419 Guyot C, Bouseta A, Scheirman V, V, Collin S. 1998. Floral Origin Markers of Chestnut and 420 Lime Tree Honeys. J Agric Food Chem 46: 625-633.

421 Hegnaur R. 1966. Chemotaxonomie der Pflanzen.

422 Hegnaur R. 1992. Benzoësaure.

423 International Organisation for Standardisation. 2007. ISO/FDIS 22855:2007 (E) Fruit and 424 vegetable products -- Determination of benzoic acid and sorbic acid concentrations -- High 425 performance liquid chromatography method.

426 Iowa State University. 1996. A user's guide to C-SIDE. Software for Intake Distribution 427 Estimation. Department of Statistics and Center for Agricultural and Rural Development; 428 Iowa State University

429 Küppers F, Jans J. 1988. Reverse-phase liquid chromatographic determination of benzoic and 430 sorbic acid acids in fresh cheese. Journal of AOAC 71: 1068-1071.

431 Matejicek D, Klejdus B, Mikes O, Sterbova D, Kuban V. 2003. Application of solid-phase 432 extraction for determination of phenolic compounds in barrique wines. Anal Bioanal Chem 433 377: 340-345.

434 Nusser SM, Carriquiry AL, Dodd KW, Fuller WA. 1996. A semiparametric transformation 435 approach to estimating usual daily intake distributions. J Am Stat Assoc 91: 1440-1449.

436 Nyman PJ, Diachenko GW, Perfetti GA, McNeal TP, Hiatt MH, Morehouse KM. 2008.

437 Survey results of benzene in soft drinks and other beverages by headspace gas

438 chromatography/mass spectrometry. J Agric Food Chem 56: 571-576. 
439 Pentilla L, Salminen S, Niemi E. 1988. Estimates on the intake of food additives in Finland. Z 440 Lebensm Unters Forsch 186: 11-15.

441 Sauh MA, Sandine WE, Ayres JW. 1990. Inhibitory effects of microgard on yoghurt and 442 cottage cheese spoilage organisms. Journal of Dairy Science 73: 387-393.

443 Sieber R, Bütikofer U, Bosset JO. 1995. Benzoic acid as a natural compound in cultured dairy 444 products and cheese. International Dairy Journal 5: 227-246.

445 Slimani N, Valsta L. 2002. Perspectives of using the EPIC-SOFT programme in the context 446 of pan- European nutritional monitoring surveys: methodological and practical implications. 447 Eur J Clin Nutr 56 Suppl 2: S63-S74.

448 Steeg E, Montag A. 1987. Nachweis aromatischer Carbonsäuren in Honig. Zeitschrift für 449 Lebensmittel-Untersuchung und -Forschung 184: 17-19.

450 Suhr KI, Nielsen PV. 2004. Effect of weak acid preservatives on growth of bakery product 451 spoilage fungi at different water activities and pH values. Int J Food Microbiol 95: 67-78.

452 Thierry A, Maillard M. 2002. Production of cheese flavour compounds derived from amino 453 acid catabolism by Propionibacterium freudenreichii. Lait 82: 17-32.

454 Van Renterghem R. 1982. Het natuurlijk benzoëzuurgehalte van yoghurt. Archives Belges 40: $455 \quad 530-538$.

456 Vermeiren L, Devlieghere F, Debevere J. 2002. Efectiveness of some recent antimicrobial 457 packaging concepts. Food additives and contaminants 19: 163-171. 
Table 1 Maximum permitted level (mg/kg or mg/l) and average concentration level (SD) (mg/kg or mg/l) of benzoic acid in different food groups FOOD GROUP

\begin{tabular}{|c|c|c|c|c|}
\hline & $\begin{array}{c}\text { Maximum } \\
\text { permitted } \\
\text { level }\end{array}$ & $\begin{array}{c}\text { Average } \\
\text { concentration level }\end{array}$ & SD & number of samples \\
\hline \multicolumn{5}{|l|}{ use of benzoic acid as an additive } \\
\hline non-alcoholic flavoured drinks & 150 & 120.5 & 17.0 & 10 \\
\hline coca cola & 150 & 128.3 & 21.7 & 3 \\
\hline lemonade & 150 & 112.1 & 10.3 & 5 \\
\hline ice-tea & 150 & 114.1 & & 1 \\
\hline aromatised water & 150 & 145.9 & & 1 \\
\hline prepared salads & 1500 & 705.0 & 205.5 & 10 \\
\hline low-sugar marmalades & 500 & $*$ & & 0 \\
\hline confectionary & 1500 & $* *$ & & 0 \\
\hline chewing gum & 1500 & $* *$ & & 0 \\
\hline non heat-treated dairy-based desserts & 300 & $* *$ & & 0 \\
\hline emulsified sauces ( $>60 \%$ fat) & 500 & $* *$ & & 0 \\
\hline emulsified sauces ( $<60 \%$ fat) & 1000 & 819.0 & 185.2 & 5 \\
\hline light sauces & 1000 & 844.6 & 7.0 & 2 \\
\hline light mayonnaise & 1000 & 498.6 & 99.2 & 2 \\
\hline dressings & 1000 & 768.6 & & 1 \\
\hline non-emulsified sauces & 1000 & 594.3 & 228.6 & 3 \\
\hline salted dried fish & 200 & $*$ & & 0 \\
\hline semi-preserved fish products & 2000 & $*$ & & 0 \\
\hline shrimps, crustaceans and molluscs & $1000^{\#}$ & $*$ & & 0 \\
\hline vegetables in vinegar/oil/brine & 2000 & $*$ & & 0 \\
\hline olives and olive-based preparations & 500 & $*$ & & 0 \\
\hline liquid soups and broth (not canned) & 500 & $*$ & & 0 \\
\hline cooked red beet & 2000 & $*$ & & 0 \\
\hline candied fruit & 1000 & $*$ & & 0 \\
\hline mustard & 1000 & $*$ & & 0 \\
\hline \multicolumn{5}{|c|}{ presence of benzoic acid as a natural substance } \\
\hline wines & & $2.5^{\&}$ & & 3 \\
\hline fruit juices & & $3.8^{\S}$ & & 3 \\
\hline yoghurts total & & & & 10 \\
\hline yoghurts with berries & & 25.7 & 6.5 & 7 \\
\hline yoghurts nature & & 24.1 & 0.2 & 3 \\
\hline butter & & $7.5^{\$}$ & & 3 \\
\hline syrups & & $3.8^{\S}$ & & 6 \\
\hline hard (Gouda) cheeses & & 13.4 & 0.5 & 2 \\
\hline cottage cheese & & $31.9^{\circ}$ & & 3 \\
\hline
\end{tabular}

SD standard deviation

*No analyses were performed because the Tier 2 showed that the contribution of these products to the total benzoic acid intake was low

** No benzoic acid was found on the labels of these products and consequently no analyses were performed \# $6000 \mathrm{mg} / \mathrm{kg}$ for grey shrimps

\& lower bound method used (3 samples below LOD) 
§ middle bound method used (for fruit juices: 2 samples below LOD and 1 sample below LOQ, for syrups 3 samples below LOD and 3 samples below LOQ)

\$ Upper bound method used (3 samples below LOQ)

${ }^{\circ}$ Only one sample above LOQ 
Table 2 The mean (SD; P50; P97.5; P99) usual consumption (g/day or ml/day) and the number of consumers (n) of the food groups in which addition of benzoic acid is permitted or in which benzoic acid is present as a natural substance (Belgian food consumption survey, 2004)

\begin{tabular}{|c|c|c|c|c|c|c|c|}
\hline \multirow[t]{2}{*}{ FOOD GROUP } & \multicolumn{7}{|c|}{ Intake (g/day or ml/day) } \\
\hline & mean & $\mathrm{SD}$ & $\mathrm{P} 50$ & P95 & $\mathrm{P} 97.5$ & P99 & $\mathrm{n}$ \\
\hline \multicolumn{8}{|l|}{ use of benzoic acid as an additive } \\
\hline non-alcoholic flavoured drinks & 228 & 251 & 164 & 719 & 884 & 1107 & 1540 \\
\hline coca cola & 140 & 201 & 23 & 545 & 673 & 846 & 975 \\
\hline lemonade & 51 & 97 & 0 & 242 & 324 & 446 & 603 \\
\hline ice-tea & 14 & 45 & 0 & 119 & 169 & 223 & 203 \\
\hline aromatised water & 23 & 82 & 0 & 174 & 277 & 417 & 211 \\
\hline prepared salads & 6 & 9 & 0 & 25 & 32 & 41 & 373 \\
\hline low-sugar marmalades & 0.6 & 3.1 & 0.0 & 0.9 & 8.9 & 16.8 & 121 \\
\hline confectionary & 1.2 & 3.6 & 0.0 & 7.7 & 11.5 & 17.4 & 256 \\
\hline chewing gum* & 0.03 & 0.87 & 0.00 & 0.00 & 0.00 & 0.00 & 15 \\
\hline non heat-treated dairy-based desserts & 16 & 17 & 11 & 49 & 60 & 77 & 166 \\
\hline emulsified sauces ( $>60 \%$ fat) & 5.8 & 4.6 & 4.8 & 14.8 & 17.6 & 21.4 & 1139 \\
\hline emulsified sauces ( $<60 \%$ fat) & 3.9 & 3.6 & 3.1 & 10.9 & 13.3 & 16.8 & 924 \\
\hline light sauces & 1.4 & 2.8 & 0.0 & 7.0 & 9.4 & 12.8 & 273 \\
\hline light mayonnaise & 0.6 & 1.1 & 0.0 & 2.8 & 3.6 & 4.7 & 254 \\
\hline dressings & 1.9 & 2.3 & 1.2 & 6.4 & 7.6 & 9.3 & 503 \\
\hline non-emulsified sauces & 17.2 & 11.1 & 15.2 & 38.4 & 44.5 & 52.5 & 1317 \\
\hline salted dried fish* & 0.1 & 3.1 & 0.0 & 0.0 & 0.0 & 0.0 & 4 \\
\hline semi-preserved fish products & 3.8 & 3.1 & 3.1 & 9.9 & 11.8 & 14.4 & 411 \\
\hline shrimps, crustaceans and molluscs & 5.3 & 5.6 & 3.9 & 16.5 & 20.0 & 24.7 & 404 \\
\hline vegetables in vinegar/oil/brine & 7.6 & 8.1 & 5.0 & 23.9 & 30.2 & 39.0 & 579 \\
\hline olives and olive-based preparations & 0.7 & 1.8 & 0.0 & 4.4 & 6.3 & 8.9 & 99 \\
\hline liquid soups and broth (not canned)* & 3.1 & 35.0 & 0.0 & 0.0 & 0.0 & 0.0 & 54 \\
\hline cooked red beet* & 0.3 & 5.8 & 0.0 & 0.0 & 0.0 & 0.0 & 29 \\
\hline candied fruit* & 0.001 & 0.038 & 0.000 & 0.000 & 0.000 & 0.000 & 1 \\
\hline mustard & 0.3 & 0.2 & 0.2 & 0.7 & 0.9 & 1.2 & 289 \\
\hline \multicolumn{8}{|c|}{ Presence of benzoic acid as a natural substance } \\
\hline wines & 67 & 95 & 20 & 260 & 320 & 402 & 885 \\
\hline fruit juices & 59 & 79 & 29 & 215 & 269 & 344 & 1072 \\
\hline yoghurts: total & 39 & 49 & 23 & 129 & 162 & 213 & 943 \\
\hline yoghurts: with fruit & 25 & 39 & 0 & 100 & 125 & 168 & 588 \\
\hline yoghurt: plane & 14 & 30 & 0 & 78 & 101 & 131 & 440 \\
\hline butter & 7 & 12 & 1 & 33 & 43 & 58 & 1164 \\
\hline syrups & 3 & 17 & 0 & 20 & 41 & 77 & 124 \\
\hline hard (gouda) cheeses & 14 & 11 & 12 & 36 & 42 & 51 & 1568 \\
\hline cottage cheeses & 11 & 26 & 0 & 67 & 92 & 124 & 429 \\
\hline
\end{tabular}

The average usual intakes are weighted and adjusted for the age and sex distribution of the Belgian population 2004 and adjusted for interview day and season The total number of consumers $(n)$ is 3083

* Average intake of the two interview days in stead of usual intake (Nusser method could not be applied due to low number of observations), not adjusted for the age and sex distribution of the Belgian population, neither for interview day and season 
Table 3. The mean estimated usual intake (mg/kg bw/day) (SD, \% contribution of food groups to the benzoic acid intake, P50, P95, P97.5,P99, \% contribution of . food groups to the ADI) of benzoic acid in the Belgian population via intake of different food groups, the Tier 2 approach

\section{$\underline{\underline{\text { FOOD GROUP }}}$}

\begin{tabular}{|c|c|c|c|c|c|c|c|c|}
\hline & mean & $\underline{\mathrm{SD}}$ & $\underline{\%}$ & $\underline{\mathrm{P} 50}$ & $\underline{\mathrm{P} 95}$ & $\underline{\mathrm{P} 97.5}$ & $\underline{\mathrm{P} 99}$ & $\%$ from $\mathrm{ADI}$ \\
\hline non-alcoholic flavoured drinks & $\underline{0.50}$ & $\underline{0.31}$ & $\underline{31.1}$ & $\underline{0.35}$ & $\underline{1.59}$ & $\underline{1.96}$ & $\underline{2.47}$ & $\underline{10.0}$ \\
\hline coca cola & $\underline{0.30}$ & $\underline{0.44}$ & $\underline{18.6}$ & $\underline{0.05}$ & $\underline{1.18}$ & $\underline{1.46}$ & $\underline{1.85}$ & $\underline{6.0}$ \\
\hline lemonade & $\underline{0.11}$ & $\underline{0.22}$ & $\underline{6.8}$ & $\underline{0.00}$ & $\underline{0.54}$ & $\underline{0.74}$ & $\underline{1.03}$ & $\underline{2.2}$ \\
\hline ice-tea & $\underline{0.03}$ & $\underline{0.10}$ & $\underline{1.9}$ & $\underline{0.00}$ & $\underline{0.27}$ & $\underline{0.38}$ & $\underline{0.50}$ & $\underline{0.6}$ \\
\hline aromatised water & $\underline{0.05}$ & $\underline{0.19}$ & $\underline{3.1}$ & $\underline{0.00}$ & $\underline{0.40}$ & $\underline{0.64}$ & $\underline{0.97}$ & $\underline{1.0}$ \\
\hline prepared salads & $\underline{0.13}$ & $\underline{0.20}$ & $\underline{8.1}$ & $\underline{0.00}$ & $\underline{0.54}$ & $\underline{0.68}$ & $\underline{0.88}$ & $\underline{2.6}$ \\
\hline low-sugar marmalades & $\underline{0.004}$ & $\underline{0.023}$ & $\underline{0.3}$ & $\underline{0.000}$ & $\underline{0.007}$ & $\underline{0.063}$ & $\underline{0.121}$ & $\underline{0.1}$ \\
\hline confectionary & $\underline{0.07}$ & $\underline{0.08}$ & 4.4 & $\underline{0.00}$ & $\underline{0.17}$ & $\underline{0.26}$ & $\underline{0.39}$ & $\underline{1.4}$ \\
\hline chewing gum* & $\underline{0.00073}$ & $\underline{0.02321}$ & $\underline{0.05}$ & $\underline{0.00000}$ & $\underline{0.00000}$ & $\underline{0.00000}$ & $\underline{0.00000}$ & $\underline{0.02}$ \\
\hline non heat-treated dairy-based desserts & $\underline{0.0690}$ & $\underline{0.0760}$ & $\underline{4.3}$ & $\underline{0.0450}$ & $\underline{0.2190}$ & $\underline{0.2750}$ & $\underline{0.3530}$ & $\underline{1.4}$ \\
\hline emulsified sauces ( $>60 \%$ fat) & $\underline{0.0420}$ & 0.0340 & 2.6 & 0.0340 & $\underline{0.1080}$ & $\underline{0.1300}$ & 0.1600 & 0.8 \\
\hline emulsified sauces ( $<60 \%$ fat $)$ & $\underline{0.0570}$ & $\underline{0.0530}$ & $\underline{3.5}$ & $\underline{0.0440}$ & $\underline{0.1590}$ & $\underline{0.1950}$ & $\underline{0.2440}$ & $\underline{1.1}$ \\
\hline $\begin{array}{l}\text { light sauces } \\
\end{array}$ & $\underline{0.0190}$ & $\underline{0.0370}$ & $\underline{1.2}$ & $\underline{0.0000}$ & $\underline{0.0950}$ & $\underline{0.1250}$ & $\underline{0.1680}$ & $\underline{0.4}$ \\
\hline light mayonnaise & 0.0089 & 0.0143 & $\underline{0.6}$ & $\underline{0.0000}$ & $\underline{0.0380}$ & $\underline{0.0485}$ & $\underline{0.0630}$ & 0.2 \\
\hline dressings & $\underline{0.0280}$ & $\underline{0.0340}$ & $\underline{1.7}$ & $\underline{0.0170}$ & $\underline{0.0950}$ & $\underline{0.1140}$ & $\underline{0.1390}$ & $\underline{0.6}$ \\
\hline non-emulsified sauces & $\underline{0.2600}$ & $\underline{0.1700}$ & $\underline{16.2}$ & $\underline{0.2240}$ & $\underline{0.5940}$ & $\underline{0.6970}$ & $\underline{0.8330}$ & $\underline{5.2}$ \\
\hline salted dried fish* & $\underline{0.00018}$ & $\underline{0.00710}$ & $\underline{0.01}$ & $\underline{0.00000}$ & 0.00000 & $\underline{0.00000}$ & $\underline{0.00000}$ & $\underline{0.004}$ \\
\hline semi-preserved fish products & $\underline{0.111}$ & $\underline{0.096}$ & $\underline{6.9}$ & $\underline{0.086}$ & $\underline{0.302}$ & $\underline{0.365}$ & $\underline{0.449}$ & $\underline{2.2}$ \\
\hline shrimps, crustaceans and molluscs & $\underline{0.110}$ & $\underline{0.150}$ & $\underline{6.8}$ & $\underline{0.066}$ & $\underline{0.404}$ & $\underline{0.521}$ & $\underline{0.689}$ & $\underline{2.2}$ \\
\hline vegetables in vinegar/oil/brine & 0.213 & 0.220 & 13.2 & 0.147 & 0.657 & 0.823 & 1.050 & 4.3 \\
\hline olives and olive-based preparations & $\underline{0.0050}$ & $\underline{0.0122}$ & $\underline{0.3}$ & $\underline{0.0000}$ & $\underline{0.0304}$ & $\underline{0.0423}$ & $\underline{0.0585}$ & $\underline{0.1}$ \\
\hline liquid soups and broth (not canned)* & $\underline{0.023}$ & $\underline{0.280}$ & $\underline{1.4}$ & $\underline{0.000}$ & $\underline{0.000}$ & $\underline{0.000}$ & $\underline{0.000}$ & $\underline{0.5}$ \\
\hline cooked red beet* & $\underline{0.010}$ & $\underline{0.170}$ & $\underline{0.6}$ & $\underline{0.000}$ & $\underline{0.000}$ & $\underline{0.000}$ & $\underline{0.000}$ & $\underline{0.2}$ \\
\hline candied fruit* & $\underline{5.449 \mathrm{E}-06}$ & $\underline{0.00043}$ & $\underline{0.0}$ & $\underline{0.00000}$ & $\underline{0.00000}$ & $\underline{0.00000}$ & $\underline{0.00000}$ & $\underline{0.0}$ \\
\hline mustard & $\underline{0.0039}$ & $\underline{0.0037}$ & $\underline{0.2}$ & $\underline{0.0029}$ & $\underline{0.0104}$ & $\underline{0.0137}$ & $\underline{0.0191}$ & $\underline{0.1}$ \\
\hline TOTAL (\% from ADI) & $\underline{1.58(31.6)}$ & $\underline{0.95}$ & & $\underline{1.39(27.8)}$ & $\underline{3.42(64.8)}$ & $\underline{3.97(79.4)}$ & $\underline{4.72(94.4)}$ & \\
\hline
\end{tabular}

\section{Deleted: ng}

Deleted: estimated usual intake

(mg/kg bw/day) 
The average usual intakes are weighted and adjusted for the age and sex distribution of the Belgian population 2004 and adjusted for interview day and season The total number of consumers (n) is 3083

*Average in stead of usual intake (due to low number of observations), not adjusted for the age and sex distribution of the Belgian population, neither for interview

day and season
Deleted: consumption days $(\mathbf{N})$ is

6166 and the total number of 
Table 4. The mean estimated usual benzoic acid intake ( $\mathrm{mg} / \mathrm{kg}$ bw/dav) (SD, P50, \% contribution of food groups to benzoic acid intake, P95, P97.5, P99, \% contribution of food groups to ADI) as an additive (97.3\%) and as a natural substance (2.7\%) in the Belgian population via intake of different food groups, the Tier 3 approach

FOOD GROI

Benzoic acid as additi

$\underline{\text { non-alcoholic flavoured drinks }}$

coca cola

lemonade

ice-tea

aromatised water

prepared salads

low-sugar marmalades

emulsified sauces $(>60 \%$ fat $) *$

emulsified sauces $(<60 \%$ fat $)$

-light sauces

- light mayonnaise

-dressings

non-emulsified sauces

$\underline{\text { salted dried fish** }}$

semi-preserved fish products

Shrimps, crustaceans, molluscs

vegetables in vinegar/oil/brine

olives and olive-based preparations

soups and broth (not canned)**

cooked red beet**

candied fruit**

$\underline{\text { mustard }}$

mean $\underline{\mathrm{SD}}$ $\underline{\%}$ $\underline{\mathrm{P} 50}$ $\underline{\mathrm{P} 95}$ $\underline{\mathrm{P} 97.5}$ $\underline{\text { P99 }}$ \% from ADI

\begin{tabular}{|c|c|c|c|c|c|c|c|}
\hline$\underline{0.40}$ & $\underline{0.53}$ & $\underline{34.3}$ & $\underline{0.28}$ & $\underline{1.28}$ & $\underline{1.58}$ & $\underline{1.99}$ & $\underline{8.0}$ \\
\hline$\underline{0.240}$ & $\underline{0.350}$ & $\underline{20.6}$ & $\underline{0.040}$ & $\underline{0.950}$ & 1.180 & $\underline{1.490}$ & $\underline{4.8}$ \\
\hline$\underline{0.090}$ & $\underline{0.180}$ & $\underline{7.7}$ & $\underline{0.000}$ & $\underline{0.433}$ & $\underline{0.591}$ & $\underline{0.828}$ & $\underline{1.8}$ \\
\hline$\underline{0.025}$ & $\underline{0.081}$ & $\underline{2.1}$ & $\underline{0.000}$ & $\underline{0.213}$ & $\underline{0.303}$ & $\underline{0.404}$ & $\underline{0.5}$ \\
\hline$\underline{0.043}$ & $\underline{0.150}$ & $\underline{3.7}$ & $\underline{0.000}$ & $\underline{0.320}$ & $\underline{0.510}$ & $\underline{0.780}$ & $\underline{0.9}$ \\
\hline$\underline{0.059}$ & $\underline{0.095}$ & $\underline{5.1}$ & $\underline{0.000}$ & $\underline{0.252}$ & $\underline{0.320}$ & $\underline{0.414}$ & $\underline{1.2}$ \\
\hline$\underline{0.004}$ & $\underline{0.023}$ & $\underline{0.3}$ & $\underline{0.000}$ & $\underline{0.007}$ & $\underline{0.063}$ & $\underline{0.121}$ & $\underline{0.1}$ \\
\hline$\underline{0.006}$ & $\underline{0.015}$ & $\underline{0.5}$ & $\underline{0.000}$ & $\underline{0.038}$ & $\underline{0.054}$ & $\underline{0.075}$ & $\underline{0.1}$ \\
\hline$\underline{0.044}$ & $\underline{0.042}$ & $\underline{3.8}$ & $\underline{0.034}$ & $\underline{0.125}$ & $\underline{0.154}$ & $\underline{0.194}$ & $\underline{0.9}$ \\
\hline$\underline{0.0150}$ & $\underline{0.0300}$ & $\underline{1.3}$ & $\underline{0.0000}$ & $\underline{0.0780}$ & $\underline{0.1030}$ & $\underline{0.1370}$ & $\underline{0.3}$ \\
\hline$\underline{0.0044}$ & $\underline{0.0071}$ & $\underline{0.4}$ & $\underline{0.0000}$ & $\underline{0.0190}$ & $\underline{0.0242}$ & $\underline{0.0314}$ & $\underline{0.1}$ \\
\hline$\underline{0.0230}$ & $\underline{0.0270}$ & $\underline{2.0}$ & $\underline{0.0140}$ & $\underline{0.0770}$ & $\underline{0.0930}$ & $\underline{0.1140}$ & $\underline{0.5}$ \\
\hline$\underline{0.1540}$ & $\underline{0.1000}$ & $\underline{13.2}$ & $\underline{0.1330}$ & $\underline{0.3530}$ & $\underline{0.4140}$ & $\underline{0.4950}$ & $\underline{3.1}$ \\
\hline$\underline{0.00018}$ & $\underline{0.00710}$ & $\underline{0.02}$ & $\underline{0.00000}$ & $\underline{0.00000}$ & $\underline{0.00000}$ & $\underline{0.00000}$ & $\underline{0.0}$ \\
\hline$\underline{0.111}$ & $\underline{0.096}$ & $\underline{9.5}$ & $\underline{0.086}$ & $\underline{0.302}$ & $\underline{0.365}$ & $\underline{0.449}$ & $\underline{2.2}$ \\
\hline$\underline{0.110}$ & $\underline{0.150}$ & $\underline{9.4}$ & $\underline{0.066}$ & $\underline{0.404}$ & $\underline{0.521}$ & $\underline{0.689}$ & $\underline{2.2}$ \\
\hline$\underline{0.213}$ & $\underline{0.220}$ & $\underline{18.3}$ & $\underline{0.147}$ & $\underline{0.657}$ & $\underline{0.823}$ & 1.050 & $\underline{4.3}$ \\
\hline$\underline{0.0050}$ & $\underline{0.0122}$ & $\underline{0.4}$ & $\underline{0.0000}$ & $\underline{0.0304}$ & $\underline{0.0423}$ & $\underline{0.0585}$ & $\underline{0.1}$ \\
\hline$\underline{0.023}$ & $\underline{0.280}$ & $\underline{2.0}$ & $\underline{0.000}$ & $\underline{0.000}$ & $\underline{0.000}$ & $\underline{0.000}$ & $\underline{0.5}$ \\
\hline$\underline{0.0103}$ & $\underline{0.1700}$ & $\underline{0.9}$ & $\underline{0.0000}$ & $\underline{0.0000}$ & $\underline{0.0000}$ & $\underline{0.0000}$ & $\underline{0.2}$ \\
\hline$\underline{0.00001}$ & $\underline{0.00043}$ & $\underline{0.0}$ & $\underline{0.00000}$ & $\underline{0.00000}$ & $\underline{0.00000}$ & $\underline{0.00000}$ & $\underline{0.0}$ \\
\hline 0.0039 & 0.0037 & $\underline{0.3}$ & 0.0029 & 0.0104 & 0.0137 & $\underline{0.0191}$ & $\underline{0.1}$ \\
\hline
\end{tabular}

Deleted: ng

Deleted: ng

Deleted: estimated usual intake

$(\mathrm{mg} / \mathrm{kg}$ bw/day) of benzoic acid 
Benzoic acid as natural substance

\begin{tabular}{|c|c|c|c|c|c|c|c|c|}
\hline wines & $\underline{0.0023}$ & $\underline{0.0033}$ & $\underline{0.2}$ & $\underline{0.0007}$ & $\underline{0.0091}$ & $\underline{0.0113}$ & $\underline{0.0141}$ & $\underline{0.05}$ \\
\hline fruit juices & $\underline{0.0033}$ & $\underline{0.0045}$ & $\underline{0.3}$ & $\underline{0.0015}$ & $\underline{0.0121}$ & $\underline{0.0155}$ & $\underline{0.0201}$ & $\underline{0.07}$ \\
\hline yoghurts total & $\underline{0.0146}$ & $\underline{0.0191}$ & 1.3 & $\underline{0.0085}$ & $\underline{0.0490}$ & $\underline{0.0620}$ & $\underline{0.0833}$ & $\underline{0.3}$ \\
\hline -yoghurts with fruit & $\underline{0.0095}$ & $\underline{0.0161}$ & $\underline{0.8}$ & $\underline{0.0000}$ & $\underline{0.0380}$ & $\underline{0.0487}$ & $\underline{0.0679}$ & $\underline{0.2}$ \\
\hline -yoghurts plane & $\underline{0.0051}$ & $\underline{0.0108}$ & $\underline{0.4}$ & $\underline{0.0000}$ & $\underline{0.0283}$ & $\underline{0.0367}$ & $\underline{0.0483}$ & $\underline{0.1}$ \\
\hline butter & $\underline{0.00081}$ & $\underline{0.00140}$ & $\underline{0.1}$ & $\underline{0.00012}$ & $\underline{0.00359}$ & $\underline{0.00481}$ & $\underline{0.00656}$ & $\underline{0.02}$ \\
\hline syrups & $\underline{0.000048}$ & $\underline{0.000190}$ & $\underline{0.0}$ & $\underline{0.000000}$ & $\underline{0.000360}$ & $\underline{0.000610}$ & $\underline{0.000960}$ & $\underline{0.0}$ \\
\hline hard (gouda) cheeses & $\underline{0.0028}$ & $\underline{0.0021}$ & $\underline{0.2}$ & $\underline{0.0023}$ & $\underline{0.0068}$ & $\underline{0.0080}$ & $\underline{0.0096}$ & $\underline{0.06}$ \\
\hline cottage cheese & $\underline{0.0049}$ & $\underline{0.0119}$ & 0.4 & $\underline{0.0000}$ & $\underline{0.0306}$ & $\underline{0.0415}$ & $\underline{0.0559}$ & $\underline{0.01}$ \\
\hline
\end{tabular}

TOTAL (\% from ADI)

$1.25(25$.

$\begin{array}{llll}1.05(21) & 2.87(57) & 3.4(68) & 4.11(82)\end{array}$

The average usual intakes are weighted and adjusted for the age and sex distribution of the Belgian population 2004 and adjusted for interview day and season The total number of consumers (n) is 3083

** Average in stead of usual intake (The Nusser method could not be applied due to low number of observations), not adjusted for the age and sex distribution of

the Belgian population, neither for interview day and season;

*without mayonnaise because no benzoic acid was found on the labels

Confectionary, chewing gum and non heat-treated dairy-based desserts were set at $0 \mathrm{mg} / \mathrm{kg}$ because no benzoic acid was found on the labels

\section{Deleted: II}

Deleted: $\mathbb{\text { II }}$

wines

Formatted: Font: $10 \mathrm{pt}$, Bold

Formatted Table

Deleted: number of consumption

days $(\mathrm{N})$ is 6166 and the total number

Deleted: as

Deleted: 0

Deleted: there was not found any

benzoic acid on the labels 


\begin{tabular}{|c|c|c|c|c|c|c|}
\hline \multirow{2}{*}{$\begin{array}{l}\text { Page 2: [1] Deleted } \\
\text { wines }\end{array}$} & \multicolumn{3}{|c|}{ Stéfanie Vandevijvere } & \multicolumn{2}{|c|}{ 1/24/2009 3:22:00 PM } & \multirow[b]{2}{*}{0.0037} \\
\hline & 0.0023 & 0.0033 & 0.20 & 0.0000 & 0.0007 & \\
\hline fruit juices & 0.0033 & 0.0045 & 0.28 & 0.0000 & 0.0015 & 0.0051 \\
\hline yoghurts total & 0.0146 & 0.0191 & 1.25 & 0.0000 & 0.0085 & 0.0232 \\
\hline -yoghurts with fruit & 0.0095 & 0.0161 & 0.81 & 0.0000 & 0.0000 & 0.0153 \\
\hline -yoghurts plane & 0.0051 & 0.0108 & 0.44 & 0.0000 & 0.0000 & 0.0051 \\
\hline butter & 0.00081 & 0.00140 & 0.07 & 0.00000 & 0.00012 & 0.00111 \\
\hline syrups & 0.000048 & 0.000190 & 0.00 & 0.000000 & 0.000000 & 0.000000 \\
\hline hard (gouda) cheeses & 0.0028 & 0.0021 & 0.24 & 0.0012 & 0.0023 & 0.0039 \\
\hline \multirow[t]{2}{*}{ cottage cheese } & 0.0049 & 0.0119 & 0.42 & 0.0000 & 0.0000 & 0.0000 \\
\hline & \multicolumn{3}{|c|}{$\%$ as natural contaminant $=2.7 \%$} & & & \\
\hline TOTAL & $1.25(0.25)$ & $\mathbf{0 . 8 3}$ & & $\begin{array}{c}0.65 \\
(0.13) \\
\end{array}$ & $\begin{array}{c}1.05 \\
(0.21) \\
\end{array}$ & $\begin{array}{c}1.63 \\
(0.33) \\
\end{array}$ \\
\hline
\end{tabular}



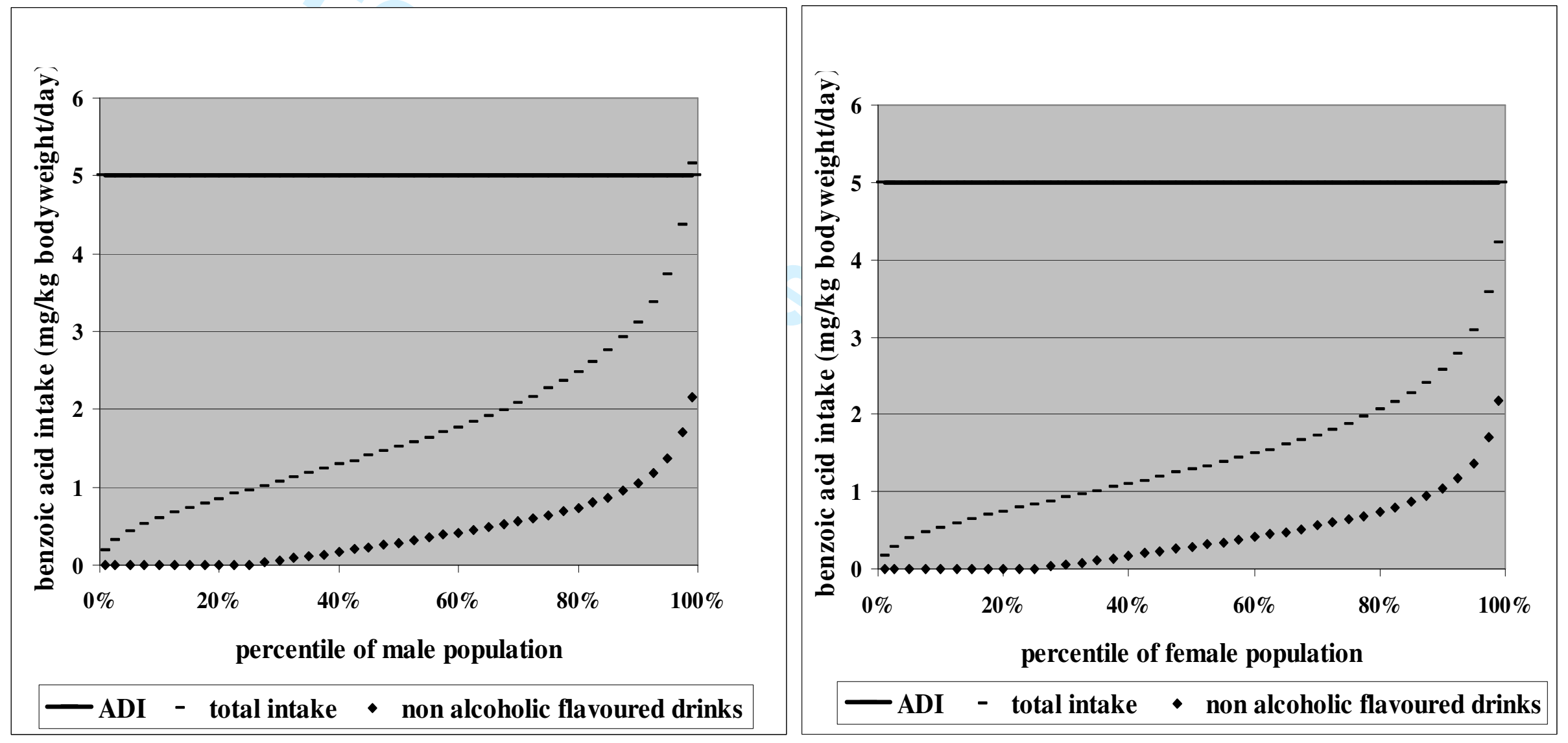

Figure 1. Cumulative distribution functions of the estimated benzoic acid intake (corrected for age) by the Belgian population (mg/kg bw/day), for men and women separately, Tier 2 approach 

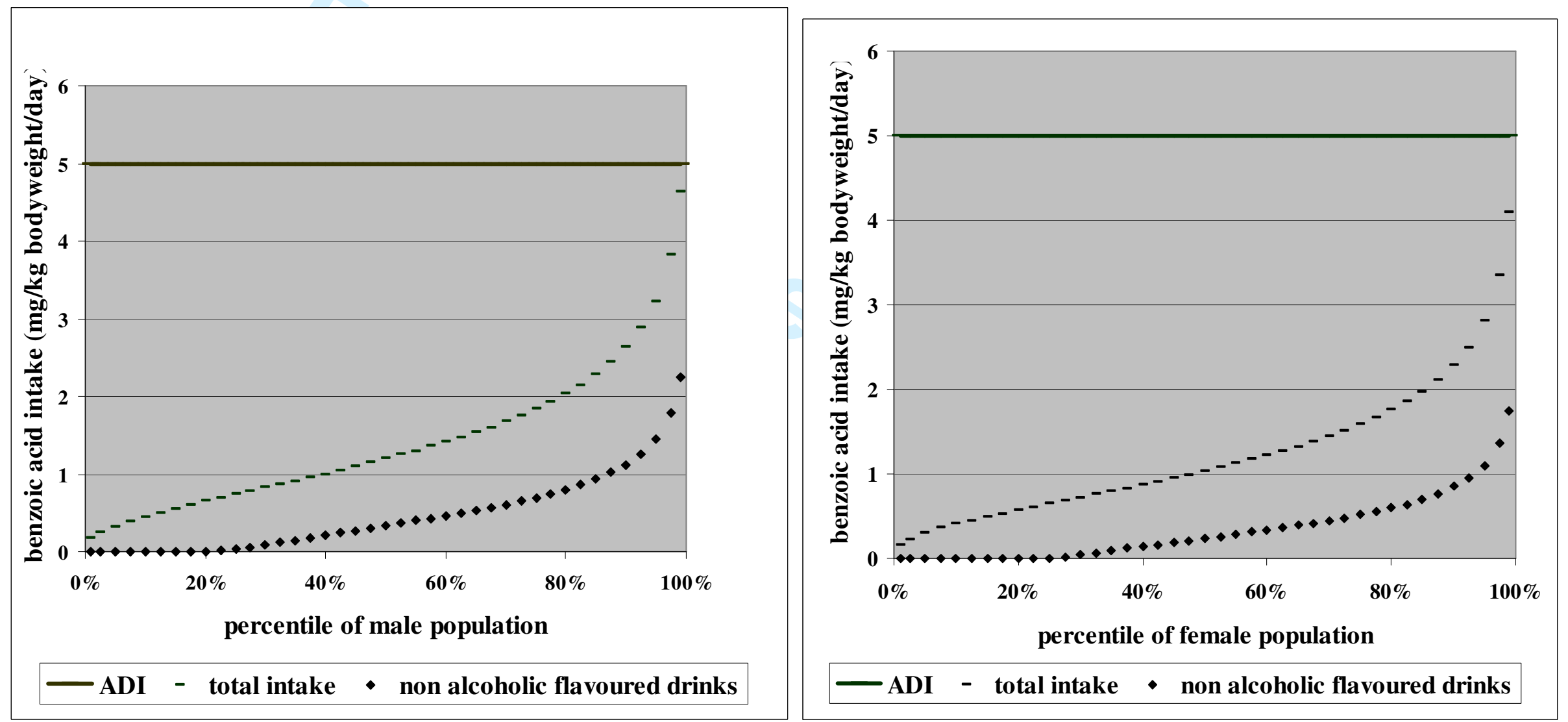

Figure 2. Cumulative distribution functions of the estimated benzoic acid intake (corrected for age) by the Belgian population (mg/kg bw/day) for men and women separately, Tier 3 approach 
